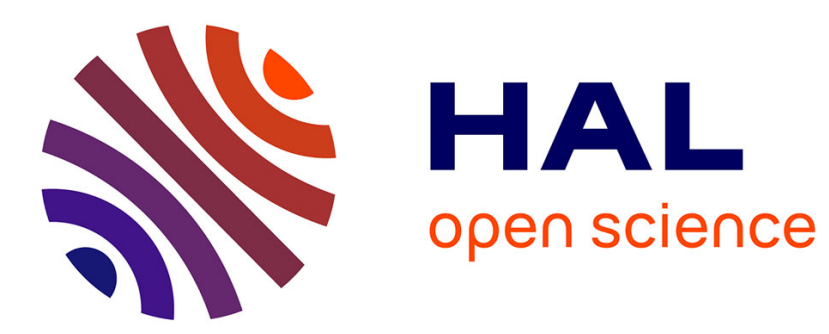

\title{
Manual asymmetries and hemispheric specialization: Insight from developmental studies \\ Hélène Cochet
}

\section{To cite this version:}

Hélène Cochet. Manual asymmetries and hemispheric specialization: Insight from developmental studies. Neuropsychologia, 2016, 93 (B), pp.335 - 341. 10.1016/j.neuropsychologia.2015.12.019 . halshs-01464518

\author{
HAL Id: halshs-01464518 \\ https://shs.hal.science/halshs-01464518
}

Submitted on 11 Jul 2017

HAL is a multi-disciplinary open access archive for the deposit and dissemination of scientific research documents, whether they are published or not. The documents may come from teaching and research institutions in France or abroad, or from public or private research centers.
L'archive ouverte pluridisciplinaire $\mathbf{H A L}$, est destinée au dépôt et à la diffusion de documents scientifiques de niveau recherche, publiés ou non, émanant des établissements d'enseignement et de recherche français ou étrangers, des laboratoires publics ou privés. 


\title{
Manual asymmetries and hemispheric specialization: Insight from developmental studies
}

\author{
Hélène Cochet \\ Laboratory CLLE-LTC (UMR 5263), University of Toulouse Jean Jaurès, Department of Psychology, 5 allées Antonio Machado, 31058 Toulouse Cedex 9, France
}

\section{A R T I C L E I N F O}

Article history:

Received 31 July 2015

Received in revised form

17 December 2015

Accepted 18 December 2015

Key words:

Handedness

Hand preference

Hemispheric specialization

Development

Children

Language acquisition

Manipulative activities

Communicative gestures

\begin{abstract}
A B S T R A C T
The objective of this review is to obtain a better understanding of the relationship between manual asymmetries and hemispheric specialization by focusing on the development of hand preference and cerebral lateralization of language. We first sought to describe the development of manual asymmetries for different activities (i.e., grasping and manipulating objects vs. communicating through gestures), and the development of cerebral asymmetries, before examining available data on the association between hand preference and HS for language. We also analyzed behavioral studies on the relation between hand preference and language development, as well as more specific studies on the relation between the cerebral control of gestures and language. Finally, we aimed at providing a wider view on functional asymmetries by emphasizing the need to study hemispheric specialization for functions other than language, and in particular for visual attention.
\end{abstract}

(c) 2015 Elsevier Ltd. All rights reserved.

\section{Introduction: on the importance of asking relevant questions}

Hemispheric specialization (HS) has evolved as a defining characteristic of humans because it may have provided them with some adaptive advantages, allowing the development of new competences by splitting functions between cerebral hemispheres (Gazzaniga, 2000). Studies on HS have long been concerned with the question of the relationship between handedness and hemispheric dominance for language, first because of the high prevalence of left HS at the population level for these two functional asymmetries (representing approximately $90 \%$ of individuals), and second because atypical HS for language in adults is more frequent in left-handers than in righthanders (Knecht et al., 2000). Focusing on the emergence of HS, especially as children acquire language and develop manual skills, has been regarded as an efficient way to further understand the relationship between these asymmetries. During the course of development, one of the objectives has thus been to find out whether children develop left HS for language as a consequence of predominantly using their right hand, or conversely.

However, the majority of left-handers present typical HS (Knecht et al., 2000) and the association between language lateralization and manual asymmetries was recently refuted in adults (Mazoyer et al., 2014), thus providing an opportunity to redefine questions on the

E-mail address: helene.cochet@univ-tlse2.fr development of behavioral and anatomo-functional asymmetries. HS for language does appear as a key feature of human cerebral organization, but such a close focus on verbal language, as a uniquely human capacity, may have relegated to the background HS for other functions, such as attention and spatial skills. As a result, relations between HS for attention and manual asymmetries have for example never been explored during development, and scarcely ever in adults. Moreover, there is now growing evidence showing that the development of handedness is a complex question that requires longitudinal measures from infancy to childhood and involving different activities, to be properly examined. The links between behavioral and cerebral asymmetries may indeed depend on some functional characteristics of the manual actions considered.

By presenting recent data, first, on the development of hand preference and second, on the development of HS, the aim of this review is to unravel the nature of the relation between manual asymmetries and language lateralization and to identify other functional asymmetries that may be of key importance for analyzing this relation.

\section{Development of handedness}

\subsection{Methodological questions}

Handedness does not entirely stabilize until adulthood, but early signs of manual asymmetries are manifest from infancy, even 
to some extent before birth, as revealed by some lateralized motor behaviors of the fetus (e.g., from 15 to 18 weeks for thumb sucking: Hepper et al., 2005). Environmental and cultural factors have been argued to strongly reinforce the initial influence of genetic and hormonal factors on the development of handedness (e.g., Fagard and Dahmen, 2004; Provins, 1992). However, one source of confusion in assessing manual asymmetries lies in the nature and the complexity of the actions observed. Rather than analyzing the causal origins of handedness, the aim of the present review is therefore to describe the development of handedness by taking into account different types of manual activities. We will focus on hand preference measures rather than on performance measures (in terms of speed and/or precision), as they may enable more relevant comparisons between infants, children and adults.

In addition to inherent difficulties in defining manual asymmetries, it is also necessary to consider methodological discrepancies across studies in the conditions under which handedness is measured and in the criteria used to categorize individuals as left-handers, right-handers or ambidextrous. In young children, direct observation of hand preference in ecological contexts (e.g., home, nursery, school) is usually preferred over the use of questionnaires assessing hand preference for daily activities (either self-report or parental questionnaires) or experimental tasks that can sometimes seem purposeless or arbitrary. Even though the two latter methods are frequently used in adults (e.g., peg-moving tasks; Annett, 1985), it is important to compare similar situations across all age ranges to analyze the development of handedness. Laterality biases in the posture of the participants and in the position of the objects involved also need to be identified to make sure that the asymmetries recorded do reflect individuals' actual preferences (e.g., Leconte and Fagard, 2004). Moreover, differences in the way of characterizing these asymmetries can constitute another obstacle to the comparisons across studies and across development: although most researchers now agree that a simple dichotomy between left- and right-handers is too limited, some choose to define intermediate categories (i.e., moderate vs. strong left- and right-handers) whereas others use handedness indices to examine both the strength and the direction of asymmetries. One of the most widely used handedness index, calculated with the formula $(R-L) /(R+L)$, where $R$ and $L$ stand for the total number of right- and left-hand responses, varies from -1 to 1 , with the sign indicating the direction of handedness and the absolute value reflecting its degree. Handedness indexes, thus reflecting the continuous distribution of hand preference, can also be used to compare the strength of manual asymmetries for different activities, independently of their direction.

\subsection{Grasping and manipulating objects}

With these methodological elements in mind, longitudinal observations over several months in infants and children have allowed researchers to identify different pathways in the development of handedness, depending on the activities considered and on individuals' patterns of HS (Cochet, 2012; Nelson et al., 2013). A first distinction has been highlighted between unimanual preference for grasping objects, which can be assessed from approximately 6 months of age, and bimanual preference for manipulating objects, with differentiated roles of the two hands: the hand regarded as non-dominant stabilizes the object for the manipulatory actions of the dominant hand (e.g., grasping a receptacle while picking up an object inserted in it). Bimanual activities usually elicit stronger degree of handedness than unimanual activities (e.g., Fagard and Marks, 2000), which may reflect the association between the emergence of new manual skills (infants become able to perform such bimanual activities by one year of age) and the need for functional specialization. However, hand preferences for unimanual and bimanual actions are not independent from each other: a longitudinal study in 6-24 montholds has shown that hand preferences for grasping objects are stable in most infants and persist across more complex manual skills acquired a few months later (Nelson et al., 2013). Other infants had no preference, but were found to shift to left or right lateralized hand use as toddlers. The majority of children were thus categorized as right-handers by 2 years of age, despite a weaker degree of preference compared to adults and a higher incidence of left-handedness. Handedness for bimanual manipulation of objects thus seems influenced by handedness for unimanual manipulation. Hand-preference strength for object manipulation has been reported to increase until 7 years of age (McManus et al., 1988), in concert with an increase in the complexity of the actions children come to perform, although preferences are less consistent and stabilize later in left-handers than in right-handers (Michel et al., 2014).

Another study, confirming consistency in hand preference from childhood to older adulthood, has also emphasized the influence of end-goal and spatial demands of the task by showing stronger right-hand preference when the objective of unimanual grasp is to eat, rather than place or manipulate the target (Gonzalez et al., 2015). Similar results have been described in 1 - to 5 -year-old children: right-hand preference for grasp-to-eat movement was present earlier (from 1 year of age) and was then stronger than hand preference for grasp-to-build movement (Sacrey et al., 2013). However, although mechanical requirements of the different types of grasp do seem identical, investigations of hand kinematics have shown smaller grip apertures during the pre-shaping phase of right-hand grasp-to-eat action, compared to other grasping movements (Flindall and Gonzalez, 2014). Smaller grip apertures are typically associated with greater precision, in relation with increased need for visual and somatosensory guidance of the hand, which might explain the greater right-hand bias for reachto-eat behavior. This right-hand advantage for grasp-to-eat actions has been argued to stem from selective pressures favoring more efficient food retrieval and consumption, thus maximizing individual fitness (e.g., Flindall and Gonzalez, 2013). Altogether, these results highlight the importance of defining end-state goals of actions for the analysis of hand preference, as they may be supported by several distinct neural networks (Gonzalez et al. 2015).

\subsection{Communicating through gestures}

Moreover, the distinction based on the nature of the activities considered has led researchers, especially in the last 15 years, to compare asymmetries for non-communicative activities (unimanual and bimanual manipulative activities) and for communicative gestures. Infants start using their hands to communicate intentionally around the end of their first year, for example by pointing toward a specific object, person or event to direct the attention of the adult. Even if the development of handedness for communicative gestures also involves a high degree of inter-individual variability, preferential use of the right hand to produce such gestures has been observed from infancy. At least before 3 years of age, these right-sided asymmetries were shown to be stronger than asymmetries characterizing unimanual or bimanual manipulative activities (Jacquet et al., 2012; Vauclair and Imbault, 2009), just like signed gestures produced by children born to deaf parents are more right-handed than other manual activities (Bonvillian et al., 1997). Moreover, several studies have shown a lack of correlation (e.g., Cochet and Vauclair, 2010b; Esseily et al., 2011), and even negative correlations (Cochet, 2012), between handedness for manipulative activities and handedness for communicative gestures. From 3 years of age, as children are more and 
more strongly lateralized, correlations between the two types of activities become significant, though moderate. A correlation coefficient of .55 was for example reported between handedness indices for reaching actions and pointing gestures in a study with 48 children aged 3-5 years (Cochet et al., 2015), a correlation that is comparable to the ones described in adults (Cochet and Vauclair, 2012, 2014). Direction of handedness was generally consistent across the two types of activities (mean handedness indexes were not found to differ), although variations were reported in the strength of hand preference. However, a more complex picture emerges when distinguishing between right-handers and nonright-handers (including both left-handers and children showing no preference): the former were found to be strongly lateralized for both communicative gestures and non-communicative reaching actions, whereas the latter, strongly left-handed for reaching actions, predominantly used their right hand for pointing gestures and symbolic gestures (Cochet and Byrne, 2015; Cochet et al., 2015). By contrast, left-handed adults were shown to preferentially use their left hand to produce pointing gestures in similar contexts, i.e., contexts in which spontaneous behaviors are recorded (Cochet and Vauclair, 2012).

Altogether, these results suggest that (1) the development of handedness follows distinct trajectories depending on whether the hands are used to act on an object or to communicate with a social partner, and (2) after approximately 3 years of age, this difference may gradually fade away or become more difficult to identify. Indeed, hand lateralization strongly increases in the course of development, for the majority of children in favor of the right hand. This may lead to a ceiling effect and therefore make comparisons between activities more difficult. Left-handers usually show less clear-cut preference, thus allowing researchers to further investigate trajectories in the development of handedness depending on the nature of the action performed. Recruiting large samples of left-handers constitutes a real challenge though, and so far we still lack developmental data that would include measures of hand preference for both non-communicative and communicative activities in a substantial number of both right-handed and lefthanded children. Focusing on atypical hand lateralization seems all the more important as researchers seek to shed light on the relationship between handedness and HS for language or other functions (see Section 3.2).

Finally, results from recent studies suggest that the distinction between different types of communicative gestures, each fulfilling specific functions, may be necessary to decipher the processes involved in the development of handedness. Although the terminology used varies across researchers (see Guidetti and Nicoladis (2008)), the classification of gestures is usually based on the nature of the relationship between the gesture and its referent: deictic gestures are directly connected to and influenced by their referents (e.g., pointing), whereas symbolic gestures have an arbitrary and convention-based relationship with their referents ( $e$. g., nodding the head, waving "goodbye") and iconic gestures illustrate specific properties of their referents (e.g., similarity in shape, size or movement of objects). The two latter categories are also sometimes referred to as representational gestures (e.g., Crais et al., 2004; Liszkowski, 2008). Moreover, there have been several studies describing various functions of pointing, highlighting differences for example in hand shape, body posture, and eye gaze accompanying the gestures (Cochet et al., 2014; Cochet and Vauclair, 2010a). Imperative pointing, used to request an object or a specific action, has been opposed to declarative pointing, produced with the intention to share some interest about an object or event with the other (declarative expressive pointing) or to provide useful information and thus help a partner (declarative informative pointing, Tomasello et al., 2007). Some variations in hand preference have been shown depending on the type of gestures produced: declarative pointing seem to elicit stronger degree of asymmetries than imperative pointing in infants (Cochet and Vauclair, 2010b) and adults (Cochet and Vauclair, 2014), and in children, only hand preference for pointing gestures and not for symbolic gestures, was found to correlate with hand preference for reaching actions (Cochet et al., 2015).

These differences suggest that the development of handedness depends on the communicative function of the gestures produced, in addition to the nature of the activity considered (i.e., manipulative $v s$. communicative actions). More studies are now required to further interpret these results, in relation to analyses of HS for language (see Section 3.2).

\section{Relationship between handedness and HS for language}

\subsection{Development of cerebral asymmetries}

Functional and structural hemispheric asymmetries have been described by means of anatomical and functional imaging studies in healthy populations (involving methodologies such as Near InfraRed Spectroscopy, functional Magnetic Resonance Imaging, Electroencephalography, or Magnetoencephalography) and in clinical studies investigating for example the effects of cerebral damage (e.g., Molfese and Molfese, 1983). Typical HS represents approximately $90 \%$ of the population (Knecht et al., 2000), but atypical forms of HS for language do exist in healthy individuals, from lack of lateralization (ambilateral individuals) to mirrored HS (i.e., rightward lateralization of language functions, see Mazoyer et al. (2014)). Evidence has accumulated showing that HS starts to develop in the first few months of life (e.g., Friederici, 2011). In particular, leftward asymmetries have been reported from infancy for receptive and expressive language functions (e.g., DehaeneLambertz et al., 2002; Dubois et al., 2009; Mills et al., 1993; see Paquette et al. (2015) for a fNIRS study focusing on language production), even if at birth, the existence of strong inter-hemispheric connectivities was shown to contrast with the adult pattern of prevalent intra-hemispheric connectivities (Perani et al., 2011). Hemispheric specialization for language then continuously increases during childhood (e.g., Friederici, 2011; Karunanayaka et al., 2010; Ressel et al., 2008). Similar developmental increase has been observed in rightward lateralization for visuo-spatial attention (Everts et al., 2009), but there is no evidence so far for a direct relationship between right hemisphere and left hemisphere lateralization in children. In adults, this relation appears to depend on HS patterns: hemispheric biases for verbal and visual functions were shown to be independent in right-handers (Powell et al., 2011; Whitehouse and Bishop, 2009), whereas a negative correlation was reported between the lateralization of these two functions in left-handers (Cai et al., 2013).

Recent advances in non-invasive techniques (e.g., fNIRS) that can be used in infants may provide researchers with opportunities to conduct more studies and fully understand how the neural basis of HS develops, especially in relation to the emergence of handedness.

\subsection{Relations between hand preference and HS for language}

In adults, there is no direct association between HS for language and either handedness or handedness strength, since between $70 \%$ and $80 \%$ of left-handers show typical language lateralization (Knecht et al., 2000; Mazoyer et al., 2014). However, few studies have investigated the relationship between hand preference and HS by taking into account several manual activities and several cerebral functions. By observing hand use for precision grasping in comparison with self-reported handedness 
questionnaires and tests of grip force and finger tapping speed, Gonzalez and Goodale (2009) have shown that language lateralization - assessed with dichotic listening task - was positively related to hand preference for precision grasping, although the correlation explained only $15 \%$ of the variance in HS. No other measure of handedness was found to be related to language lateralization. The comparison of different handedness measures during development (between 6 and 16 years of age) has also revealed a significant relationship between language lateralization - assessed with functional transcranial Doppler ultrasound - and hand preference on a reaching task, compared to self-reported inventory and measure of hand skill in a peg-moving task (Groen et al., 2013). The correlation was nevertheless weak, explaining $8 \%$ of the variance in language lateralization when examining handedness strength, and $19 \%$ when including only right-handers. The authors emphasized in their conclusion the considerable independence between handedness and language lateralization, although this relationship could not be analyzed in left-handers due to their small number in the sample studied. Results of a study involving a large sample of adults balanced for handedness have shown that handedness and language lateralization were significantly associated only in strongly-atypical individuals, who represent less than $1 \%$ in the general population (Mazoyer et al., 2014). These individuals exhibited strong right hemisphere dominance for both language and hand use.

Very few similar studies are available in children, but a recent event-related potential analysis in 18- and 24-month-olds also failed to show direct causal relationship between handedness for grasping actions and language lateralization (Fagard et al., 2014). Moreover, all these studies are limited to measures of handedness for manipulative activities, i.e., purely motor functions, whereas above-mentioned evidence suggests that communicative functions of movement affect hand choice. The relation between HS and hand preference for gestures therefore deserves to be investigated on its own.

\subsection{Relations between hand preference and language development}

The lack of neuroimaging data in relation to the asymmetry of communicative gestures has been counterbalanced by an increasing number of studies focusing on the development of communication in infants and children, with the objective of determining whether hand preference for gestures develops in association with language acquisition. First, infants who are righthanded for pointing gestures were found to understand and produce more words than non-right-handed pointers (Esseily et al., 2011). The degree of right-hand preference for pointing also increases as language abilities develop in toddlers categorized as good language learners (from the calculation of a developmental quotient for language: Vauclair and Cochet, 2013). Second, the lexical spurt - a strong and rapid growth in lexical production occurring around 18 months - is associated with an increase in right-hand preference for pointing (Cochet et al., 2011). These results suggest that common cerebral resources are allocated simultaneously to the development of communicative gestures and language in young children. By contrast, the studies listed above did not show any relationship between language acquisition and handedness for either unimanual or bimanual manipulative activities, which, in addition to the absence of correlation between the asymmetry of both types of activities (see Section 2.3), may emphasize the distinction between two typically left-lateralized systems: (1) a system specialized in both vocal and gestural communication, and (2) a system controlling non-communicative manual movements. Measures of hand preferences for pointing and symbolic gestures in children between 3 and 5 years of age were not found to significantly correlate with language level, but a negative correlation was reported between the strength of hand preference for reaching actions and language score (Cochet and Byrne, 2015; Cochet et al., 2015), which might further emphasize the distinction between the two previously mentioned systems.

Moreover, differentiating between several lateralization patterns may be necessary to further understand the exact nature of the relationship between hand preference and language. Consistent right-hand preference for reaching and object manipulation during infancy, assessed at monthly intervals from 6 to 14 months, was shown to be associated with advanced language skills measured around 10 months later, whereas infants with an inconsistent preference and who became right-handed or left-handed as toddlers had typical language scores (Nelson et al., 2014). Consistent left-hand preference is generally too scarce before 1 year of age, which has not allowed researchers to identify patterns of language development that might be specific to those infants. Direct measures of HS in 4-year-olds, using functional transcranial Doppler ultrasound, have also revealed a lack of language lateralization at the group level in children considered as late-talkers when first seen at 20 months of age, whereas children without impaired language showed a pronounced left-hemisphere lateralization (Bishop et al., 2014). Although it was not predictive of language difficulties at the individual level, atypical lateralization may therefore constitute a marker of risk for language impairment.

However, sample size in developmental studies do not usually allow for a distinction taking into account both hand preference (left- and right-handers) and HS (typical and atypical language lateralization). Further investigation of the relationship between hand preference and language development may also require distinguishing between language production and comprehension and between different functions of communicative gestures. In particular, informative pointing gestures have been suggested to play a key role in the development of left-hemisphere specialization for language (Cochet and Vauclair, 2010a), as they are characterized by (1) a strong right-sided asymmetry from early on (see Section 2.3) and (2) a close relationship with language abilities in terms of the cognitive skills they involve (Liszkowski et al., 2006).

To summarize, the study of hand preference in relation with language acquisition can provide some insights into the development of left-hemispheric specialization, provided the nature of the actions observed is taken into account. The asymmetry of movements serving communicative functions might thus constitute an indicator of cerebral lateralization for of language.

\subsection{Relations between cerebral control of gestures and language}

Gestural communication plays a central role in the development of language, allowing infants to engage in social interactions and express meanings they are not yet able to verbalize (e.g., Colonnesi et al., 2010; Rowe and Goldin-Meadow, 2009). The association between gestures and language has also been investigated through the analysis and comparison of their respective neural correlates. Semantic information conveyed through speech and gestures are integrated simultaneously by the brain (Özyürek et al., 2007) and common networks in left-lateralized inferior frontal and posterior temporal regions are activated by the perception of speech and symbolic gestures (e.g., He et al., 2015; Willems et al., 2007; Xu et al., 2009). By applying the N400 paradigm, event-related potential studies have shown that the processing of gestures (including deictic, symbolic, and iconic gestures) involved linguistic brain areas in adults (Proverbio et al., 2015). In infants, N400 congruency effect was reported in a priming task for pictures preceded by both words and gestures, demonstrating that words and gestures elicit similar patterns of semantic activation (Sheehan et al., 2007). 
The comprehension of gestures in typical population elicits mechanisms closer to the ones involved in sign language processing by non-hearing speakers. Left hemisphere lateralization has been described in deaf adults for sign language processing (e.g., Grossi et al., 1996) and in deaf children exposed to cued speech, which is a visual mode of communication using handshapes in combination with the mouth movements of speech, to represent the phonemes of a spoken language. Deaf participants and hearing controls were also shown to display comparable left hemisphere specialization for semantic processing of written language (D'Hondt and Leybaert, 2003), and similar accuracy of phonological representations (Leybaert, 2000), suggesting that semantic and phonological abilities develop independently of the modality (acoustic versus visual) through which language is perceived. Moreover, the production of signs activates regions similar to those implicated in spoken language use, including Broca's area ( $e$. g., Corina et al., 2003; Emmorey et al., 2007). The study of sign language thus offers further support to the existence of a common neural system for gestural and verbal communication (Gentilucci and Dalla Volta, 2008).

Moroever, the association between cerebral control of speech and communicative gestures has been highlighted in studies demonstrating the influence of gestures on voice parameters (e.g., Krahmer and Swerts, 2007). Voice frequency spectrum was shown to increase when a word and the corresponding gesture are produced simultaneously, compared to conditions involving only the production of words, or involving both modalities but meaningless arm movements and pseudo-words (Bernardis and Gentilucci, 2006). Changes in the acoustic properties (F2 formant) of vocalizations have also been reported when infants produce request gestures towards large objects, in comparison with small objects (Bernardis et al., 2008). However, this effect on the vocal spectra was also observed for object grasping, in both infants and adults (see Gentilucci and Dalla Volta (2007), for a review), thus revealing the influence of the size of grasped objects - and corresponding changes in hand shape - on lip opening kinematics and voice parameters, an influence that seems independent from the communicative nature of movements. As a consequence, the relation between language and gesture might be considered as one particular form of the relation between language and action, which has been supported by the existence of neurons controlling grasping movements of both hand and mouth (e.g., Willems and Hagoort, 2007). In the perspective of action-grounded cognition, it could thus be argued that the relation between right-handedness and left HS for language relies on individuals' situated perceptualmotor abilities (without necessarily implying the existence of a causal relation between hand preference and HS). These abilities may gradually allow the construction of action-grounded representations of both manual and vocal-motor activities across development (e.g., Anderson et al., 2012). Such representations may involve action in general rather than being restricted to communicative gestures. From a basic mirror system for grasping, Arbib (2011) has proposed a sequence of sensorimotor development in which manual skills and gestures, along with the evolution of more and more complex imitation skills, would form the basis of speech movements and proto-language. Entangled brain mechanisms for praxis and language in adults may reflect the interrelated roles of pantomime, tool making and skill transmission in the course of human evolution (see also Corballis (2010)).

A comparable developmental sequence has been proposed in infancy, starting with unimanual reaching and developing into role-differentiated bimanual manipulation, which, through increasing tool-using skills and social learning mechanisms, might facilitate the development of language processing (Michel et al., 2013). However, the differences described earlier between hand preferences for manipulative activities and communicative gestures suggest that it is an early specialization for intentional communication that may initially link the development of handedness with the development of HS for language in infancy (see Sections 2.3 and 3.3). Complex processes may thereafter come to underlie the relationship between language, action, and gesture in adults. These processes now deserve further investigation, which necessitates considering distinct lateralization patterns and, possibly, HS for functions other than language.

\section{Relationship between handedness and HS for spatial attention}

Studies on the relationships between behavioral and anatomofunctional asymmetries have mainly concentrated on HS for language, partly due to historical definitions of language as a unique and "sacred" human quality. However, studying HS of other functions such as visuo-spatial attention, non-verbal communication and emotional processing (without being exhaustive) is necessary to gain a better understanding of cerebral functional organization, i.e., to determine whether handedness is more directly associated to functions other than language, and to address the issue of co-lateralization of some functions like language and visuo-spatial attention (e.g., Mellet et al., 2013).

A strong rightward asymmetry in the ventral network for spatial attention was recently described in adults, irrespective of hand-preference strength, whereas an association was shown between left-handedness and rightward asymmetry of the dorsal frontoparietal attention network (Petit et al., 2015). This association was even stronger in left-handers with a right-sighting eye, which might involve, in addition to the advantage of having the same hemisphere controlling the dominant hand and attention processes, an advantage in the visual exploration of the spatial environment. The authors have therefore proposed that HS for spatial attention develops from manipulo-spatial coordination, but this question has not yet been directly addressed in children, and few studies in adults have investigated the relationship between handedness and HS for spatial attention.

Moreover, a difference was reported in left-handers depending on language lateralization patterns: in individuals with typical lateralization (i.e., left HS for language), cerebral activation during a visuospatial attention task was most pronounced in the right hemisphere, whereas it was most pronounced in the left hemisphere in individuals with atypical lateralization (i.e., right HS for language; Willems et al., 2014, see also Cai et al. (2013)). These results may constitute evidence in favor of a co-lateralization of linguistic and visuospatial functions, although it may not apply to right-handers (Powell et al., 2011; Whitehouse and Bishop, 2009). Analyzing HS for attention may therefore help understand why the relationship between handedness and HS for language seems to be indirect, by revealing some possible intermediate and complementary processes controlling the development of HS. This might be true for other lateralized cognitive and/or emotional functions, but the mechanisms linked to visual attention, by allowing mapping a spatial context onto perceptuo-motor activities, may be closely intertwined with both language and manual actions, including gestures and especially pointing (see Section 3.4).

\section{Conclusion}

Even if the variety of methods used to measure manual asymmetries and analyze HS has led to some discrepancies across studies (see Cochet and Byrne (2013)), it is clear that right-handedness and left hemispheric dominance for language are largely prevalent in the population, characterizing about $90 \%$ of 
individuals. Such asymmetries have been regarded as essential aspects of the emergence of tool-making skills and language in human evolution, but the relationship between hand preference and language lateralization is far from being fully understood. In the present review, we aimed to decipher the complexity of this relationship by focusing first on the development of manual asymmetries, in relation with language acquisition, and then on the development of HS for language, in relation with functional brain asymmetries associated with gestural communication and visual attention.

Studies presented in the different sections of this review have revealed several points of key importance to analyze the relationship between handedness and HS: (1) the nature of the activities considered, including both the manual skills they require and the functions they serve, and (2) individual lateralization patterns for hand use and for language dominance. In particular, we have reported recent evidence that increase in the degree of hand preference for gestures is associated with increase in toddlers' language abilities, and that the asymmetry of communicative gestures develops independently from the asymmetry of non-communicative manual actions, at least before 3 years of age. The question of a causal link between predominant use of the right hand and the development of left HS for language may thus appear irrelevant, or too limited: the association between these two asymmetries may rather rely on cerebral networks common to gestures and language, which have been identified with neuroimaging techniques in adults. The association with hand preference for other manual actions may then involve a complex multimodal integration, which accompanies increasing specialization during development.

Moreover, the analysis of left-handers, and especially those with atypical lateralization, is necessary to gain a deeper insight into the relationship between handedness and HS. It will also be important to compare different HS patterns from infancy to adulthood in order to characterize the dynamics of this relationship in the course of development, and thus determine whether these dynamics are associated with the emergence of specific sensorimotor skills and/or cognitive abilities. For this purpose, studying HS in atypical development - language disorders, but also autism spectrum disorders - and in psychiatric pathologies may also provide rich information on anatomo-functional brain organization.

\section{References}

Anderson, M.L., Richardson, M.J., Chemero, A., 2012. Eroding the boundaries of cognition: implications of embodiment. Top. Cogn. Sci. 4, 717-730.

Annett, M., 1985. Left, Right, Hand and Brain: The Right Shift Theory. Lawrence Erlbaum Associates, London.

Arbib, M.A., 2011. From mirror neurons to complex imitation in the evolution of language and tool use. Annu. Rev. Anthropol. 40, 257-273.

Bernardis, P., Bello, A., Pettenati, P., Stefanini, S., Gentilucci, M., 2008. Manual actions affect vocalizations of infants. Exp. Brain Res. 184, 599-603.

Bernardis, P., Gentilucci, M., 2006. Speech and gesture share the same communication system. Neuropsychologia 44 (2), 178-190.

Bishop, D.V., Holt, G., Whitehouse, A.J., Groen, M., 2014. No population bias to lefthemisphere language in 4-year-olds with language impairment. PeerJ 2, e507.

Bonvillian, J.D., Richards, H.C., Dooley, T.T., 1997. Early sign language acquisition and the development of hand preference in young children. Brain Lang. 58, 1-22.

Cai, Q., Van der Haegen, L., Brysbaert, M., 2013. Complementary hemispheric specialization for language production and visuospatial attention. Proc. Natl. Acad. Sci. USA 110, E322-E330.

Cochet, H., 2012. Development of hand preference for object-directed actions and pointing gestures: a longitudinal study between 15 and 25 months of age. Dev. Psychobiol. 54, 105-111.

Cochet, H., Byrne, R.W., 2013. Evolutionary origins of human handedness: evaluating contrasting hypotheses. Anim. Cogn. 16, 531-542.

Cochet, H., Byrne, R.W., 2015. Communication in the second and third year of life: relationships between nonverbal social skills and language. Manuscript submitted for publication.
Cochet, H., Centelles, L., Jover, M., Plachta, S., Vauclair, J., 2015. Hand preferences in preschool children: reaching, pointing and symbolic gestures. Laterality: Asymmetries Body Brain Cogn. 20, 501-516.

Cochet, H., Jover, J., Oger, L., Vauclair, J., 2014. Morphological differences between imperative and declarative pointing: hand shape, arm extension and body posture. J. Mot. Behav. 46, 223-232.

Cochet, H., Jover, M., Vauclair, J., 2011. Hand preference for pointing gestures and bimanual manipulation around the vocabulary spurt period. J. Exp. Child. Psychol. 110, 393-407.

Cochet, H., Vauclair, J., 2010a. Features of spontaneous pointing gestures in toddlers. Gesture $10(1)$, 86-107.

Cochet, H., Vauclair, J., 2010b. Pointing gestures produced by toddlers from 15 to 30 months: different functions, hand shapes and laterality patterns. Infant Behav. Dev. 33, 432-442.

Cochet, H., Vauclair, J., 2012. Hand preferences in human adults: noncommunicative actions vs. communicative gestures. Cortex 48, 1017-1026.

Cochet, H., Vauclair, J., 2014. Deictic gestures and symbolic gestures produced by adults in an experimental context: hand shapes and hand preferences. Laterality: Asymmetries Body Brain Cogn. 19, 278-301.

Colonnesi, C., Stams, G., Koster, I., Noom, M.J., 2010. The relation between pointing and language development: a meta-analysis. Dev. Rev. 30 (4), 352-366.

Corballis, M.C., 2010. Mirror neurons and the evolution of language. Brain Lang. 112 (1), 25-35.

Corina, D.P., San Jose-Robertson, L., Guillemin, A., High, J., Braun, A.R., 2003. Language lateralization in a bimanual language. J. Cognit. Neurosci. 15 (5), 718-730.

Crais, E., Day-Douglas, D., Cox-Campbell, C., 2004. The intersection of the development of gestures and intentionality. J. Speech Lang. Hear. Res. 47 (3), 678-694.

Dehaene-Lambertz, G., Dehaene, S., Hertz-Pannier, L., 2002. Functional neuroimaging of speech perception in infants. Science 298, 2013-2015.

D’Hondt, M., Leybaert, J., 2003. Lateralization effects during semantic and rhyme judgement tasks in deaf and hearing subjects. Brain Lang. 87, 227-240.

Dubois, J., Hertz-Pannier, L., Cachia, A., Mangin, J.F., Le Bihan, D., Dehaene-Lambertz, G., 2009. Structural asymmetries in the infant language and sensori-motor networks. Cereb. Cortex 19 (2), 414-423.

Emmorey, K., Mehta, S., Grabowski, T.J., 2007. The neural correlates of sign versus word production. NeuroImage 36, 202-208.

Esseily, R., Jacquet, A.-Y., Fagard, J., 2011. Handedness for grasping objects and pointing and the development of language in 14-month-old infants. Laterality 16 (5), 565-585.

Everts, R., Lidzba, K., Wilke, M., Kiefer, C., Mordasini, M., Schroth, G., Perrig, W. Steinlin, M., 2009. Strengthening of laterality of verbal and visuospatial functions during childhood and adolescence. Hum. Brain Mapp. 30, 473-483.

Fagard, J., Dahmen, R., 2004. Cultural influences on the development of lateral preferences: a comparison between French and Tunisian children. Laterality 9 67-78.

Fagard, J., Marks, A., 2000. Unimanual and bimanual tasks and the assessment of handedness in toddlers. Dev. Sci. 3 (2), 137-147.

Fagard, J., Sirri, L., Rämä, P., 2014. Effect of handedness on the occurrence of semantic N400 priming effect in 18- and 24-month-old children. Front. Psychol. $5,1-8$.

Flindall, J.W., Gonzalez, C.L.R., 2013. On the evolution of handedness: evidence for feeding biases. PLoS One 8 (11), e78967.

Flindall, J.W. Gonzalez C.L.R. 2014. Eating interrupted: the effect of intent on handto-mouth actions. J. Neurophysiol. 112, 2019-2025.

Friederici, A.D., 2011. The brain basis of language processing: from structure to function. Physiol. Rev. 91 (4), 1357-1392.

Gazzaniga, M.S., 2000. Cerebral specialization and interhemispheric communication: does the corpus callosum enable the human condition? Brain 123 , 1293-1326.

Gentilucci, M., Dalla Volta, R., 2007. The motor system and the relationships between speech and gesture. Gesture 7, 159-177.

Gentilucci, M., Dalla Volta, R., 2008. Spoken language and arm gestures are controlled by the same motor control system. Q. J. Exp. Psychol. 61, 944-957.

Gonzalez, C.L.R., Flindall, J.W., Stone, K.D., 2015. Hand preference across the lifespan: effects of end-goal, task nature, and object location. Front. Psychol. 5, 1579.

Gonzalez, C.L.R., Goodale, M.A., 2009. Hand preference for precision grasping predicts language lateralization. Neuropsychologia 47 (14), 3182-3189.

Groen, M.A., Whitehouse, A.J., Badcock, N.A., Bishop, D.V., 2013. Associations be tween MP and cerebral lateralisation for language: a comparison of three measures in children. PLoS One 8, e64876.

Grossi, G., Semenza, C., Corazza, S., Volterra, V., 1996. Hemispheric specialization for sign language. Neuropsychologia 34 (7), 737-740.

Guidetti, M., Nicoladis, E., 2008. Introduction to special issue: gestures and communicative development. First Lang. 28, 107-115.

He, Y., Gebhardt, H., Steines, M., Sammer, G., Kircher, T., Nagels, A., Straube, B., 2015 The EEG and fMRI signatures of neural integration: an investigation of meaningful gestures and corresponding speech. Neuropsychologia 72, 27-42.

Hepper, P.G., Wells, D.L., Lynch, C., 2005. Prenatal thumb sucking is related to postnatal handedness. Neuropsychologia 43 (3), 313-315.

Jacquet, A.-Y., Esseily, R., Rider, D., Fagard, J., 2012. Handedness for grasping objects and declarative pointing: a longitudinal study. Dev. Psychobiol. 54 (1), 36-46.

Karunanayaka, P., Schmithorst, V.J., Vannest, J., Szaflarski, J.P., Plante, E., Holland, S K. 2010. A group independent component analysis of covert verb generation in children: a functional magnetic resonance imaging study. NeuroImage 51, 
$472-487$

Knecht, S., Dräger, B., Deppe, M., Bobe, L., Lohmann, H., Flöel, A., Ringelstein, B.E. Henningsen, H., 2000. Handedness and hemispheric language dominance in healthy humans. Brain 123, 2512-2518.

Krahmer, E., Swerts, M., 2007. The effects of visual beats on prosodic prominence: acoustic analyses, auditory perception and visual perception. J. Mem. Lang. 57 (3), 396-414

Leconte, P., Fagard, J., 2004. Influence of object spatial location and task complexity on children's use of their preferred hand depending on their handedness consistency. Dev. Psychobiol. 45, 51-58.

Leybaert, J., 2000. Phonology acquired through the eyes and spelling in deaf children. J. Exp. Child Psychol. 75, 291-318.

Liszkowski, U., 2008. Before L1: a differentiated perspective on infant gestures. Gesture 8 (2), 180-196.

Liszkowski, U., Carpenter, M., Striano, T., Tomasello, M., 2006. Twelve- and 18month-olds point to provide information for others. J. Cogn. Dev. 7, 173-187.

Mazoyer, B., Zago, L., Jobard, G., Crivello, F., Joliot, M., Perchey, G., Mellet, E., Petit, L. Tzourio-Mazoyer, N., 2014. Gaussian mixture lodeling of hemispheric lateralisation for language in a large sample of healthy individuals balanced for handedness. PLoS One 9, e101165.

McManus, I.C., Sik, G., Cole, D.R., Mellon, A.F., Wong, J., Kloss, J., 1988. The development of handedness in children. Br. J. Dev. Psychol. 6, 257-273.

Mellet, E., Jobard, G., Zago, L., Crivello, F., Petit, L., Joliot, M., Mazoyer, B., TzourioMazoyer, N., 2013. Relationships between hand laterality and verbal and spatial skills in 436 healthy adults balanced for handedness. Laterality 19, 1-22.

Michel, G.F., Babik, I., Nelson, E.L., Campbell, J.M., Marcinowski, E.C., 2013. How the development of handedness could contribute to the development of language. Dev. Psychobiol. 55, 608-620.

Michel, G.F., Babik, I., Sheu, C. -F., Campbell, J.M., 2014. Latent classes in the developmental trajectories of infant handedness. Dev. Psychol, 50, 349-359.

Mills, D.L., Coffey-Corina, S.A., Neville, H.J., 1993. Language acquisition and cerebral specialization in 20-month-old infants. J. Cogn. Neurosci. 5, 317-334.

Molfese, D.L., Molfese, V.J., 1983. Hemispheric lateralization in infancy. In: Young, G. (Ed.), Manual Specialization and the Developing Brain: Longitudinal Research. Academic Press, New York

Nelson, E.L., Campbell, J.M., Michel, G.F., 2013. Unimanual to bimanual: tracking the development of handedness from 6 to 24 months. Infant Behav. Dev. 36 (2) $181-188$.

Nelson, E.L., Campbell, J.M., Michel, G.F., 2014. Early handedness in infancy predicts language ability in toddlers. Dev. Psychol. 50, 809-814.

Özyürek, A., Willems, R.M., Kita, S., Hagoort, P., 2007. On-line integration of semantic information from speech and gesture: insights from even-related brain potentials. J. Cogn. Neurosci. 19, 605-616.

Paquette, N., Lassonde, M., Vannasing, P., Tremblay, J., González-Frankenberger, B., Florea, O., Béland, R. Lepore, F., Gallagher, A., 2015. Developmental patterns of expressive language hemispheric lateralization in children, adolescents and adults using functional near-infrared spectroscopy. Neuropsychologia 68 117-125.

Perani, D., Saccuman, M.C., Scifo, P., Anwander, A., Spada, D., Baldoli, C., Poloniato, A., Lohmann, G., Friederici, A.D., 2011. Neural language networks at birth. Proc. Natl. Acad. Sci. USA 108, 16056-16061.

Petit, L., Zago, L., Mellet, E., Jobard, G., Crivello, F., Joliot, M., Mazoyer, B., TzourioMazoyer, N., 2015. Strong rightward lateralization of the dorsal attentional network in left-handers with right sighting-eye: an evolutionary advantage. Hum. Brain Mapp. 36, 1151-1164.

Powell, J.L., Kemp, G.J., García-Finaña, M., 2011. Association between language and spatial laterality and cognitive ability: an fMRI study. Neurolmage 59, 1818-1829.

Proverbio, A.M., Gabaro, V., Orlandi, A., Zani, A., 2015. Semantic brain areas are involved in gesture comprehension: an electrical neuroimaging study. Brain Lang. 147, 30-40.

Provins, K.A., 1992. Early infant asymmetries and handedness: a critical evaluation of the evidence. Dev. Neuropsychol. 8 (4), 325-365.

Ressel, V., Wilke, M., Lidzba, K., Lutzenberger, W., Krägeloh-Mann, I., 2008. Increases in language lateralization in normal language as observed using magnetoencephalography. Brain Lang. 106, 167-176.

Rowe, M.L., Goldin-Meadow, S., 2009. Early gesture selectively predicts later language learning. Dev. Sci. 12, 182-187.

Sacrey, L.A., Arnold, B., Whishaw, I.Q., Gonzalez, C.L.R., 2013. Precocious hand use preference in reach-to-eat behavior versus manual construction in 1- to 5-yearold children. Dev. Psychobiol. 55, 902-911.

Sheehan, E.A., Namy, L.L., Mills, D.L., 2007. Developmental changes in neural activity to familiar words and gestures. Brain Lang. 101, 246-259.

Tomasello, M., Carpenter, M., Liszkowski, U., 2007. A new look at infant pointing. Child Dev. 78, 705-722.

Vauclair, J., Cochet, H., 2013. Hand preference for pointing and language development in toddlers. Dev. Psychobiol. 55, 757-765.

Vauclair, J., Imbault, J., 2009. Relationships between manual preferences for manipulative actions and for pointing gestures in infants and toddlers. Dev. Sci. 12 . 1060-1069.

Whitehouse, A.J.O., Bishop, D.V.M., 2009. Hemispheric division of function is the result of independent probabilistic biases. Neuropsychologia 47, 1938-1943.

Willems, R.M., van der Haegen, L., Fisher, S.E., Francks, C., 2014. On the other hand: including left-handers in cognitive neuroscience and neurogenetics. Nat. Rev. Neurosci. 15, 193-201.

Willems, R.M., Hagoort, P., 2007. Neural evidence for the interplay between language, gesture and action: a review. Brain Lang. 101 (3), 278-298.

Willems, R.M., Ozyurek, A., Hagoort, P., 2007. When language meets action: the neural integration of gesture and speech. Cereb. Cortex 17 (10), 2322-2333.

Xu, J., Gannon, P.J., Emmorey, K., Smith, J.F., Braun, A.R., 2009. Symbolic gestures and spoken language are processed by a common neural system. Proc. Natl. Acad. Sci. USA 106 (49), 20664-20669. 